\title{
HD 121190: A cool multiperiodic slowly pulsating B star with moderate rotation ${ }^{\star}$
}

\author{
C. Aerts ${ }^{1,2}$ and K. Kolenberg ${ }^{1,3}$ \\ ${ }^{1}$ Instituut voor Sterrenkunde, Katholieke Universiteit Leuven, Celestijnenlaan 200 B, 3001 Leuven, Belgium \\ e-mail: conny@ster . kuleuven.ac.be \\ 2 Department of Astrophysics, University of Nijmegen, PO Box 9010, 6500 GL Nijmegen, the Netherlands \\ ${ }^{3}$ Institut für Astronomie, Universität Wien, Türkenschanzstrasse 17, 1180 Wien, Austria
}

Received 20 May 2004 / Accepted 20 October 2004

\begin{abstract}
We have gathered and analysed multicolour Geneva photometry and high-resolution spectroscopy of the stars HD 121190 (B9V) and HD 106419 (B9III) whose short-term periodic variability had become evident from their HIPPARCOS data. We find three significant frequencies for HD 121190 in the Geneva data: $2.6831,2.6199$ and $2.4713 \mathrm{~cd}^{-1}$ and classify the star as a slowly pulsating B star, the coolest single star of that class known to date. Its amplitude ratios are compatible with lowdegree gravity modes. The spectra reveal low-amplitude variability and $v \sin i=118 \mathrm{~km} \mathrm{~s}^{-1}$ which implies that the star rotates at $26 \%$ of its critical velocity. For HD 106419 we are unable to confirm the period found from the HIPPARCOS photometry in our ground-based data; rather we find a marginally significant frequency of $0.8986 \mathrm{c} \mathrm{d}^{-1}$. This star is probably an evolved slowly pulsating B star with complex variations. HD 106409 is also a moderate rotator as the spectra reveal $v \sin i=78 \mathrm{~km} \mathrm{~s}^{-1}$, which is also $26 \%$ of its critical velocity.
\end{abstract}

Key words. stars: oscillations - stars: variables: general - stars: individual: HD 121190 - stars: individual: HD 106419 stars: early-type - line: profiles

\section{Introduction}

One of the remarkable by-products of the ESA HIPPARCOS mission was the discovery of a large amount of periodically variable B stars (Waelkens et al. 1998). Among these, the large number of newly discovered slowly pulsating B stars (hereafter abbreviated as SPBs) was quite unexpected. Large followup studies of these stars were initiated by Aerts et al. (1999) and Mathias et al. (2001). This resulted in a sample of some 25 well-known members of this class of multiperiodic gravitymode oscillators, the statistical properties of which were summarized by De Cat (2002) and seem to fulfill all expectations from theoretical studies (Pamyatnykh 1999).

Among the known SPBs, the double-lined spectroscopic binary HD 123515 stands out as the coolest one with an effective temperature of $12000 \mathrm{~K}$ and spectral type B9IV (De Cat et al. 2000; De Cat \& Aerts 2002). All other members are at least $1500 \mathrm{~K}$ hotter (De Cat 2002). Given that most of the SPBs known nowadays were discovered from the HIPPARCOS unbiased sample (Waelkens et al. 1998), we must conclude that the lower SPB instability strip is less populated than its upper counterpart and one may question if single B8/9 SPBs exist at

* Based on data gathered with the Swiss $0.7 \mathrm{~m}$ telescope equipped with the photometer P7 of the Geneva Observatory and with the FEROS spectrograph attached to the ESO $2.2 \mathrm{~m}$ telescope, both situated at La Silla in Chile. all. A similar question, albeit from a somewhat different perspective, was already tackled by Baade $(1989 a, b)$ many years before the results of HIPPARCOS became available. He performed an extensive spectroscopic search for line-profile variability among 22 late-B main-sequence stars and subgiants and came to the same null result: no B8/9 IV/V line-profile variable stars were found. B8/9 stars are also absent in the list of Be line-profile variables analysed and interpreted recently in terms of non-radial oscillations by Rivinius et al. (2003).

As a continuation of the quest for oscillating late-B stars, several stars caught our attention as they were listed in the HIPPARCOS catalogue of periodic variables with periods of only a few hours. The current paper concerns two of these stars of spectral type B9. HD 121190 (B9V, $V$ mag = 5.7) was already classified as a new candidate SPB by Waelkens et al. (1998), although the two classification schemes used by these authors did not give consistent results for this star. Waelkens et al. (1998) found one period of $0.38 \mathrm{~d}$ in the HIPPARCOS photometry for this star. All other confirmed SPBs have longer periods (De Cat \& Aerts 2002; De Cat 2002). Moreover, theoretical models typically predict periods above half a day (Pamyatnykh 1999), although this lower limit is not strict as it depends largely on metallicity and rotational effects, the latter of which are usually neglected in theoretical SPB excitaton calculations so far. A second star that struck us is HD 106419 (B9III, $V$ mag $=8.3$ ). The HIPPARCOS team assigned an 


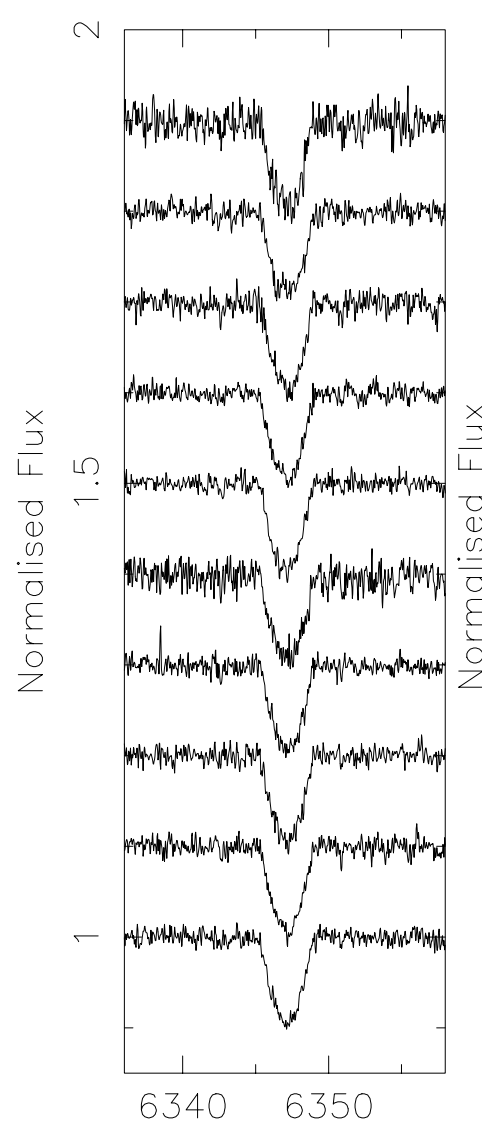

Wavelength $(\AA)$

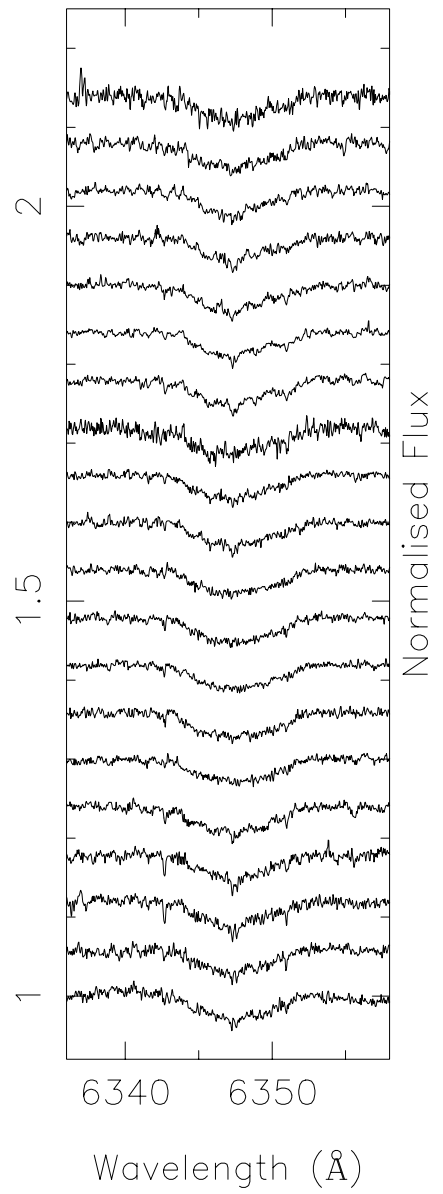

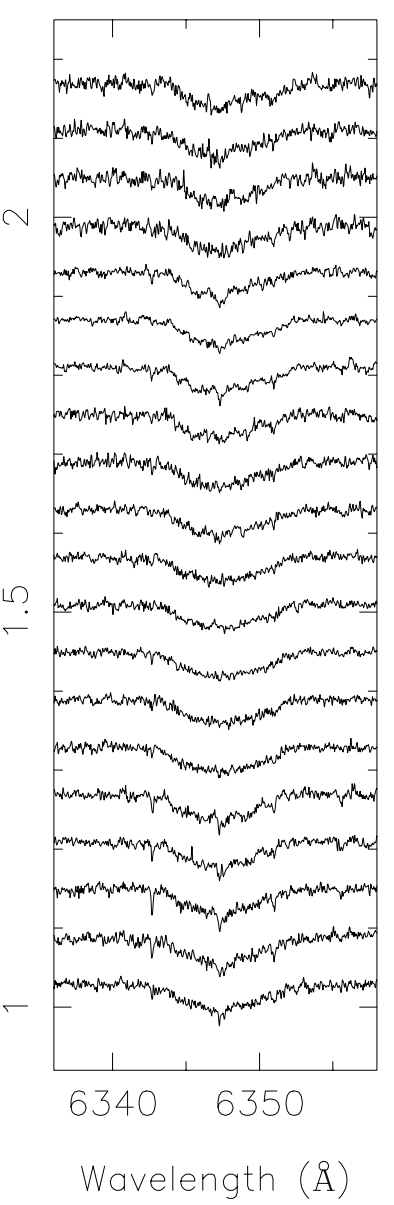

Fig. 1. The selected SiII $26347.091 \AA$ Aines in the spectra of HD 106419 (left) and HD 121190 (middle and right). Time increases from bottom to top of the panels.

intrinsic period of $0.11 \mathrm{~d}$ to it. If confirmed, such a short period would imply very severe deviation from current theoretical model predictions.

The current paper describes the results of our follow-up campaigns dedicated to HD 106419 and HD 121190. In Sect. 2 we discuss the data that were gathered with the goal to confirm their periodic variability. Section 3 deals with the determination of the basic physical parameters of these stars and their position with respect to the SPB instability strip. A detailed frequency analysis is described in Sect. 4. Finally, we discuss our findings in Sect. 5.

\section{Data description}

We included HD 106419 and HD 121190 in our long-term photometric monitoring programme of pulsating B stars of Leuven University. In this framework we obtained Geneva 7-colour high-precision photometry for the two stars during one campaign with a 3-week duration and one campaign of 9 weeks within one season. The integration times were typically $5 \mathrm{~min}$ for HD 106419 and $3 \mathrm{~min}$ for HD 121190 resulting in a precision of about $5 \mathrm{mmag}$ per measurement. In total we obtained 165 measurements for HD 106419 with a time span of 104 days and in 145 datapoints for HD 121190 with a 115 days time span. For HD 121190 we had already 5 very old Geneva measurements available, which result in an overall time span of 6922 days. The old measurements turned out to be fully compatible with the new ones, so we kept them in our analysis.

Given the confirmed photometric variability of the stars (see Sect.4), we obtained additional spectroscopic data with the FEROS spectrograph attached to the ESO $2.2 \mathrm{~m}$ telescope. The data were taken during 5 consecutive nights 2 years after the photometric campaigns. The main goal of this spectroscopic study was to exclude binarity, to check for evidence of line-profile variability, as well as to derive an accurate estimate of the rotational velocity of the stars.

The line-profile variability of the slow rotators among the SPBs is by far best studied from the SiII doublet at $\lambda 4128,4130 \AA$ (see, e.g., Aerts et al. 1999). However, for our two target stars, this doublet turned out to be blended due to their relatively high rotation rate compared to other SPBs and so we focused on the much better isolated SiII $6347.091 \AA$ line. In order not to miss any line-profile variability with short periods, we decided to integrate during $15 \mathrm{~min}$ for both stars. This resulted in a signal-to-noise ratio between 80 and 150 for HD 106419 and between 150 and 300 for HD 121190 in the region near the SiIL $6347.091 \AA$ line. We show all the data of this line, for both target stars, in Fig. 1.

A logbook of all the data is provided in Table 1. 
Table 1. Logbook of the dedicated measurements of HD 106419 and HD 121190. The Heliocentric Julian Dates (HJD) are given in days with respect to 2450000 and $N$ denotes the number of measurements.

\begin{tabular}{crrrrrr}
\hline \hline & \multicolumn{3}{c}{ Photometry } & \multicolumn{3}{c}{ Spectroscopy } \\
\hline Star & \multicolumn{2}{c}{ HJD } & $N$ & \multicolumn{2}{c}{ HJD } & $N$ \\
& Begin & End & & Begin & End & \\
\hline HD 106419 & 486.8 & 502.8 & 17 & 1270.3 & 1270.3 & 1 \\
& 541.7 & 592.6 & 148 & 1271.2 & 1271.2 & 1 \\
& & & & 1272.0 & 1272.2 & 2 \\
& & & & 1273.1 & 1273.3 & 2 \\
& & & & 1274.0 & 1274.3 & 4 \\
\hline HD 121190 & 485.8 & 503.8 & 17 & 1270.3 & 1270.4 & 7 \\
& 541.8 & 601.6 & 133 & 1271.2 & 1271.3 & 3 \\
& & & & 1272.1 & 1272.4 & 10 \\
& & & & 1273.1 & 1273.4 & 10 \\
& & & & 1274.1 & 1274.4 & 11 \\
\hline
\end{tabular}

\section{Physical parameters}

We used the method outlined by Künzli et al. (1997) to estimate the mean effective temperature and gravity from the average Geneva colours. The results are provided in Table 2 and are in very good agreement with the spectral types B9III (HD 106419) and B9V (HD 121190) listed in Simbad. We point out that the error estimates for the temperature and gravity resulting from the code by Künzli et al. are internal errors only, in the sense that they result from interpolation in the grids of standard stars. They assume the average Geneva colours and the reference tables to be error-free. The true uncertainties are typically a factor two larger and it is these more realistic error estimates that we provide in Table 2.

Subsequently, we used the photometric calibration outlined in Heynderickx et al. (1994) together with interpolation in the evolutionary models published by Schaller et al. (1992) to estimate the mass, radius, luminosity and bolometric magnitude of each star. The result of the physical parameters obtained in this way are summarised in Table 2 and are used to place the two stars in the HR diagram in Fig. 2. The SPB instability strip calculated by Pamyatnykh (1999) is also indicated, as well as some evolutionary models. The instability strip is not considered beyond the TAMS. It can be seen that HD 106419 is clearly an evolved star. HD 121190, on the other hand, is a main-sequence star situated just at the lower limit of the instability strip, which was computed for solar metallicity and ignoring core overshooting as well as rotation.

The thermal broadening of metallic lines in B9 stars amounts to only a few $\mathrm{km} \mathrm{s}^{-1}$ and is negligible compared to the measured width of the lines. For this reason, and keeping in mind the variable nature of the stars, we estimated their $v \sin i$ by averaging the full-width-at-half-maximum of all the SiII $26347.091 \AA$ lines shown in Fig. 1, i.e., $v \sin i=$ $\langle F W H M\rangle / \sqrt{\ln 16}$. This resulted in 78 and $118 \mathrm{~km} \mathrm{~s}^{-1}$ for respectively HD 106419 and HD 121190. The projected rotation velocity of HD 106419 equals the mean value found for 70 B9-B9.5 III stars (Abt et al. 2002). The mean $v \sin i$-value found by Abt et al. (2002) for 145 B9-B9.5 V stars amounts to $134 \mathrm{~km} \mathrm{~s}^{-1}$ which is only slightly higher than HD 121190 's

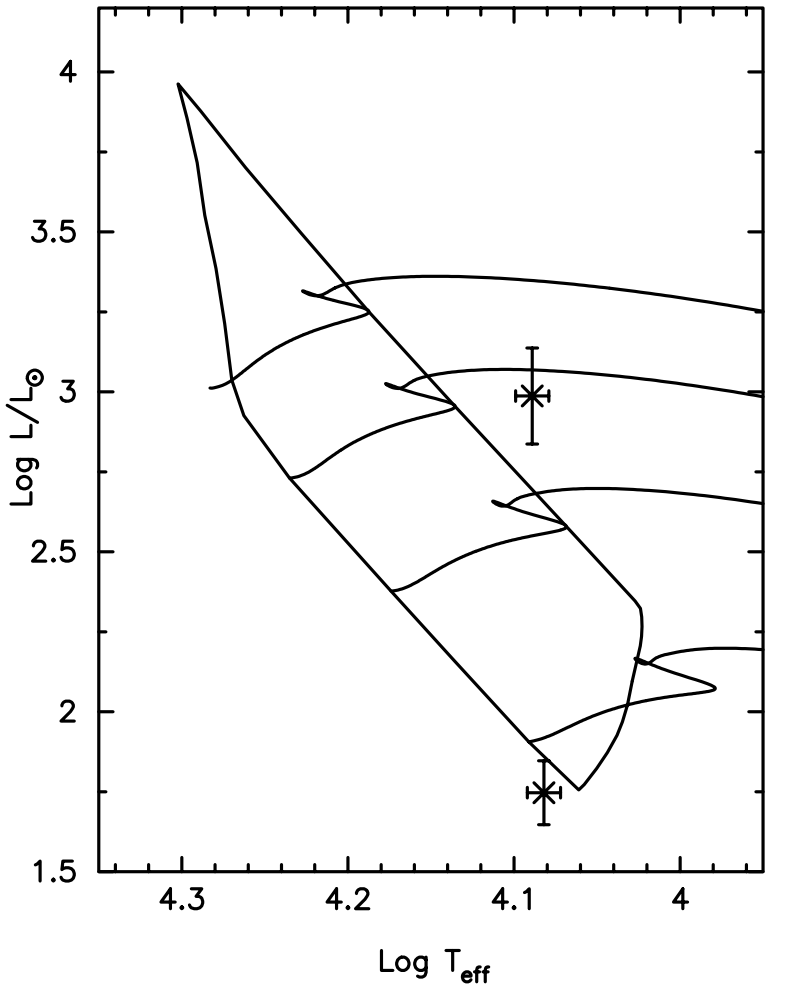

Fig. 2. The position of the two stars (crosses) with respect to the SPB instability strip which is taken from Pamyatnykh (1999) and is based on OPAL G93/21 opacities (Iglesias \& Rogers 1996) for a composition $X, Z=0.70,0.02$. Evolutionary tracks for $3,4,5$ and $6 M_{\odot}$ are also shown. Overshooting and rotation were not included in the calculation of the strip and evolutionary tracks.

value. Both our target stars are therefore "normal" rotators among the B-type stars.

Taking the values for the mass and radius given in Table 2 we determined the critical rotation velocity for both stars from the well-known expression valid within the Roche approximation for a centrally condensed star: $v_{\text {crit }}=\sqrt{2 G M / 3 R_{\text {pole }}}$ (e.g. Tassoul 1978). It is found that both HD 106419 and HD 121190 rotate at at least $26 \%$ of their critical velocity. At this low rate, the equatorial radius of the star is only a factor 1.03 larger than the polar radius such that the oblateness of the star due to the centrifugal force is negligible.

All but one of the confirmed SPBs rotate much slower than $26 \%$ of their critical velocity (De Cat 2002), HD 1976 being a notable exception with $32 \%$ (Mathias et al. 2001). From this perspective, both our target stars rotate much faster than the average SPB.

\section{Frequency analysis}

We searched for frequencies in the data with the method outlined in Scargle (1982). The accuracy of the frequencies was calculated as $\sigma_{\mathrm{f}} \sim \sigma / \sqrt{N} A T$ where the proportionality constant is of order $1, A$ is the amplitude of the frequency $f, N$ is the number of data points, $T$ is the total time base and $\sigma$ is the error estimate of the individual measurements, which is 5 mmag for the Geneva photometry (Cuypers 1987). After 
Table 2. The physical parameters of the two programme stars. The mass and radius are given in solar units; $v$ sin $i$ and $v_{\text {crit }}$ are given in km s ${ }^{-1}$.

\begin{tabular}{ccccccccc}
\hline \hline Star & $\log T_{\text {eff }}$ & $\log g$ & Mass & Radius & $\log \left(L / L_{\odot}\right)$ & $M_{\text {bol }}$ & $v \sin i$ & $v_{\text {crit }}$ \\
\hline HD 106419 & $4.089 \pm 0.010$ & $3.42 \pm 0.15$ & $4.7 \pm 0.5$ & $6.8 \pm 0.5$ & $2.99 \pm 0.15$ & $-2.71 \pm 0.15$ & $78 \pm 4$ & 297 \\
HD 121190 & $4.082 \pm 0.010$ & $4.43 \pm 0.09$ & $2.8 \pm 0.3$ & $1.7 \pm 0.3$ & $1.75 \pm 0.10$ & $0.39 \pm 0.10$ & $118 \pm 3$ & 458 \\
\hline
\end{tabular}
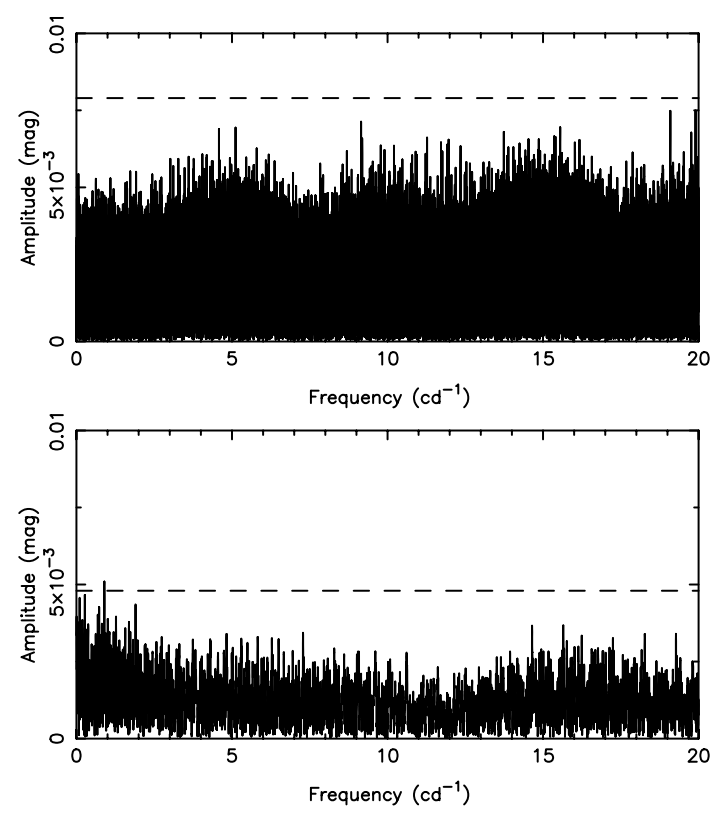

Fig. 3. The periodograms for the HIPPARCOS (upper) and Geneva $U$ (lower) data of HD 106419. The dashed horizontal line indicates the $4 \mathrm{~S} / \mathrm{N}$ ratio determined in the way explained in the text.

having derived the frequencies by a prewhitening process, we determined the signal-to-noise $(\mathrm{S} / \mathrm{N})$ ratio in the periodogram of the residuals by averaging the amplitude of all peaks for the frequency range $[0,20] \mathrm{cd}^{-1}$. We use the $4 \mathrm{~S} / \mathrm{N}$ value as a criterion to judge a frequency to be significant or not, following Breger et al. (1993).

\subsection{HD 106419}

We first of all checked the short period of $0.11 \mathrm{~d}$ given by the HIPPARCOS team for this star. In the upper panel of Fig. 3 we show the periodogram for the $\mathrm{H}_{\mathrm{p}}$ data. It can be seen that a strong frequency peak occurs at $19.09005 \mathrm{~cd}^{-1}$. The peak given in the HIPPARCOS catalogue is also present at frequency $f_{\mathrm{Hp}}=9.15074 \mathrm{c} \mathrm{d}^{-1}$. There are also two additional peaks near $5 \mathrm{c} \mathrm{d}^{-1}$. However, none of the mentioned peaks fulfills the $4 \mathrm{~S} / \mathrm{N}$ criterion which is indicated by the dashed horizontal line. The variance reduction for any of the candidate frequencies is below $14 \%$. With the adopted criterion for significance, we therefore have to reject the results from the HIPPARCOS catalogue.

In the Geneva $U$ data, for which the periodogram is shown in the lower part of Fig. 3, we do find one marginally significant frequency: $f_{U}=0.8986 \pm 0.0005 \mathrm{c} \mathrm{d}^{-1}$. The amplitude of this frequency amounts to $0.0051 \pm 0.0013 \mathrm{mag}$ and its variance reduction is $15 \%$. The value of this frequency is typical for the gravity modes expected to occur in SPBs, unlike the high frequency determined by the HIPPARCOS team. Nevertheless, it is only slightly above the $4 \mathrm{~S} / \mathrm{N}$ level. Moreover, we do not find any clear evidence of $f_{U}$, or any other frequency, in the data for the other 6 Geneva filters. From this we cannot but consider $f_{U}$ to be a candidate frequency only as long as we have no independent evidence of its reality. We show the phase diagrams for the HIPPARCOS and Geneva $U$ data, for respectively $f_{\mathrm{Hp}}$ and $f_{U}$, in Fig. 4. These phase diagrams are typical for multiperiodic stars with low amplitudes (see De Cat \& Aerts 2002, for additional examples).

The SiII $66347.091 \AA$ profiles shown in Fig. 1, while rather noisy, show small-amplitude variability of the line center. This is independent proof that the star is variable, but we have too few spectra to do an independent frequency search for these data.

Our conclusion for this star is that it is probably an evolved SPB with a complex low-amplitude frequency pattern, covering a wide range in frequency. This suggestion needs further confirmation, however.

\section{2. $H D 121190$}

The Geneva data for HD 121190 are completely in line with the HIPPARCOS results. Moreover, they clearly point towards a multiperiodic star. In Fig. 5 we summarize the results for the Geneva $U$ filter, in which the amplitudes are largest. We find evidence for three significant frequencies: $f_{1}=2.6831 \pm$ $0.0004 \mathrm{~cd}^{-1}, f_{2}=2.6199 \pm 0.0004 \mathrm{~cd}^{-1}$ and $f_{3}=2.4713 \pm$ $0.0007 \mathrm{c} \mathrm{d}^{-1}$. After prewhitening with $f_{1}, f_{2}, f_{3}$, the frequency $2.8452 \mathrm{c} \mathrm{d}^{-1}$ occurs, but it does no longer fulfill the $4 \mathrm{~S} / \mathrm{N}$ criterion. The frequency $f_{2}$ corresponds to the one found in the HIPPARCOS photometry. The phase plots for the U filter for subsequent stages of prewhitening are shown in the upper panel of Fig. 6. In that same plot we also show the phase diagram for $f_{1}$ for the colours $U-B, B-V$ as well as the HIPPARCOS data folded with $f_{2}$. The amplitudes and phases in the 7 filters for the significant frequencies are provided in Table 3 , as well as the variance reduction for a triperiodic least-squares fit with $f_{1}, f_{2}, f_{3}$ fixed.

From the amplitudes listed in Table 3 we derive the ratios with respect to the $U$ filter shown in Fig. 7. Although the error bars are large, it can be seen that a clear decrease of the amplitude from $\mathrm{U}$ to $\mathrm{B}$ occurs. This result is typically found from observations for all confirmed SPBs (De Cat \& Aerts 2002; De Cat et al. 2004). It is also what is expected from theoretical computations (Townsend 2002; Dupret et al. 2003). The observed decrease in amplitude from $U$ to $G$ is similar for all three modes, although the main mode's decrease is somewhat smaller. In general, this points towards modes with similar degree.

A more quantitative statement on the degree of the oscillation modes can in principle be derived from the confrontation 

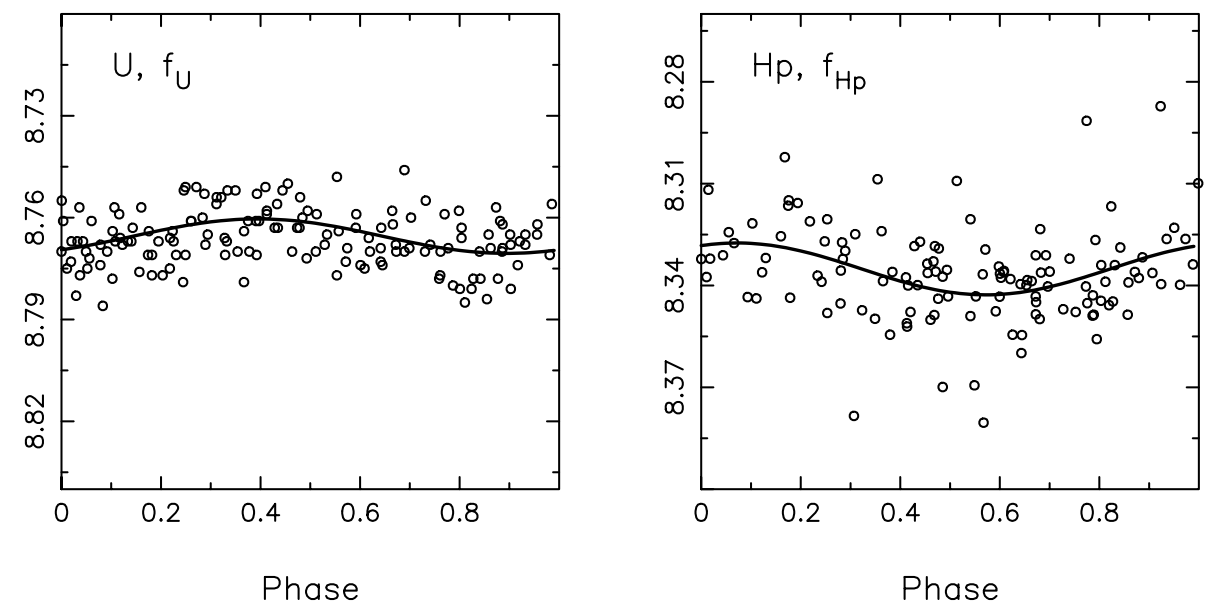

Fig. 4. The phase diagrams for the Geneva $U$ (left) and HIPPARCOS (right) data folded with different frequencies, of which the values are given in the text. The open dots are the data and the full line is a least-squares fit for the considered frequency.

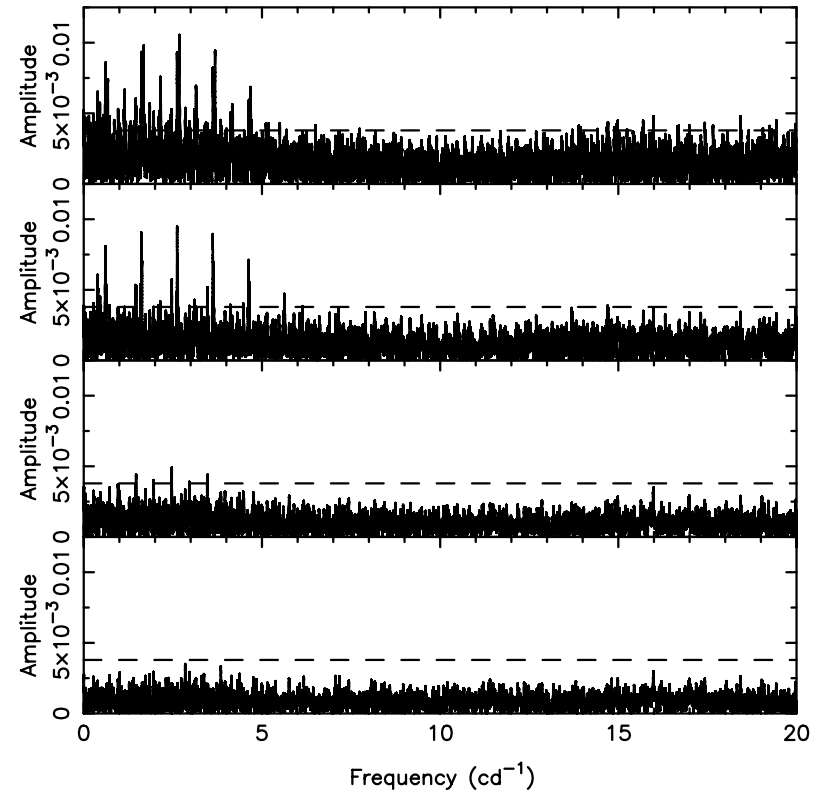

Fig. 5. The periodograms for the Geneva $U$ data of HD 121190 after subsequent stages of prewhitening with the frequencies provided in the text. The amplitudes are expressed in magnitudes. The dashed horizontal line indicates the $4 \mathrm{~S} / \mathrm{N}$ ratio determined in the way explained in the text.

of the observed ratios with those predicted from model calculations. Townsend (2002) has provided a complete systematic study of the photometric behaviour expected for SPBs in the approximation of non-rotating stars. In his Fig. 9, he provides the amplitude and phase differences predicted by models for all unstable $\ell=1, \ldots, 4$ modes in the SPB instability strip as well as the observed values found by De Cat \& Aerts (2002). For mode identification in specific stars, however, one has to consider only the ratios and phase differences for models with appropriate physical parameters. The top rows in Figs. 7 and 8 of Townsend (2002) are suitable to identify the three detected modes of HD 121190. First of all we point out that none of the $\ell=3$ and 4 modes are found to be unstable for the appropriate mass. This result is confirmed by us on the basis of the entirely independently written evolution and pulsation codes CLÉS and MAD (see Dupret et al. 2003). This leaves us with the possibility of $\ell=1$ or 2 , if we take the theoretical excitation predictions based on the $\kappa$ mechanism applied to models with a solar mixture for granted - see, however, Pamyatnykh et al. (2004) and Ausseloos et al. (2004) for an example where such predictions fail, albeit for a pressure mode in the much hotter $\beta$ Cep star $v$ Eri. The confrontation of our observed amplitude ratios and very small phase differences with the theoretical predictions by Townsend (2002) leads to the conclusion that the dominant mode must be $\ell=1$. We are unable to discriminate between $\ell=1$ and 2 for the two additional modes.

An important remark that has to be made regarding the achieved mode identification is the fact that the Coriolis force plays a significant role in the behaviour of gravity modes of low frequency whenever the pulsational frequency in the corotating frame becomes larger than half the rotational frequency (Townsend 2003, and references therein). In such a situation, neglect of the Coriolis force in mode identification from amplitude ratios is inappropriate (see Fig. 5 in Townsend 2003). We have, unfortunately, no means to evaluate the importance of the Coriolis force in the treatment of HD 121190's oscillations because we have no information about its inclination angle $i$. In any case, from the radius estimate listed in Table 2 and the measured $v \sin i$, we deduce $\Omega \sin i=1.37 \mathrm{~cd}^{-1}$, which is about half of the measured oscillation frequencies. It is therefore quite likely that the frequencies observed for HD 121190 are significantly shifted with respect to those in a non-rotating star due to the Coriolis force, unless we see the star almost equator-on. In the latter case our mode identification is still accurate, but for smaller inclination angles our results have to be treated with caution.

Due to the moderate rotation of HD 121190 it is not obvious from the lines shown in Fig. 1 whether profile variability occurs and if so, whether it is consistent with the photometric variability. In order to check that, we computed line diagnostics for the 40 profiles shown in Fig. 1. We considered the equivalent width, the radial velocity and the first three velocity moments in the definition by Aerts et al. (1992). The time span of the 

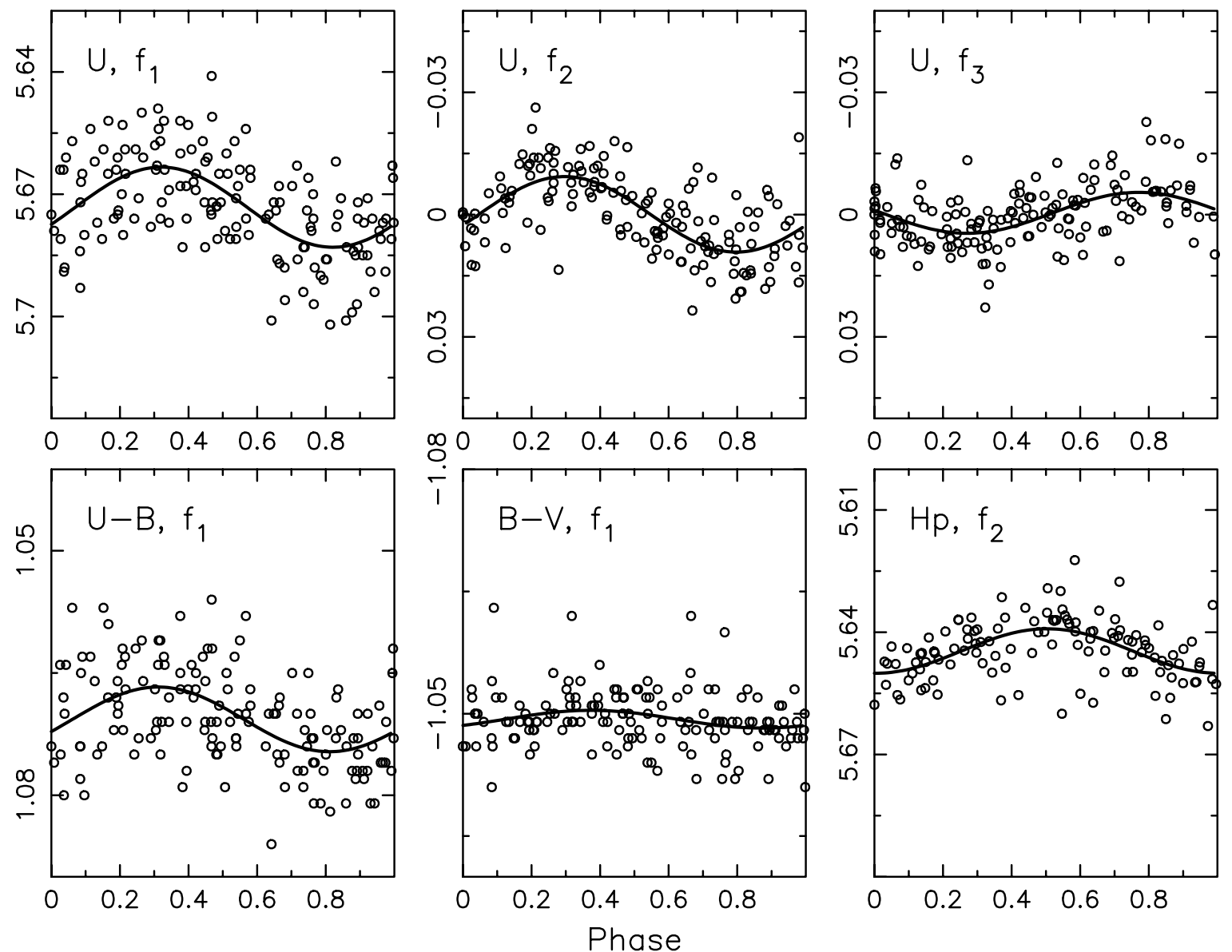

Fig. 6. Upper panels: the phase diagrams for the Geneva $U$ data of HD 121190 for the three significant frequencies $f_{1}, f_{2}, f_{3}$ after subsequent stages of prewhitening. Lower panels: left and middle: the Geneva colours $U-B$ and $B-V$ folded with the main frequency; right: the HIPPARCOS data folded with $f_{2}$. The open dots are the data and the full line is a least-squares fit for the indicated frequency.

Table 3. Results of harmonic fits to the Geneva lightcurves of HD 121190. A stands for the amplitude, expressed in millimag, and $\phi$ for the phase, expressed in $2 \pi$ radians. The adopted reference epoch for $\phi=0.0$ corresponds to HJD 2450000.0 .

\begin{tabular}{ccccccccc}
\hline \hline & & $U$ & $B_{1}$ & $B$ & $B_{2}$ & $V_{1}$ & $V$ & $G$ \\
\hline$f_{1}$ & $\mathrm{~A}$ & $9.7 \pm 1.0$ & $6.3 \pm 0.8$ & $5.8 \pm 0.8$ & $5.9 \pm 0.8$ & $4.5 \pm 0.8$ & $4.8 \pm 0.7$ & $4.3 \pm 0.8$ \\
& $\phi$ & $0.814 \pm 0.016$ & $0.822 \pm 0.021$ & $0.822 \pm 0.021$ & $0.806 \pm 0.023$ & $0.839 \pm 0.030$ & $0.812 \pm 0.024$ & $0.820 \pm 0.030$ \\
$f_{2}$ & $\mathrm{~A}$ & $8.9 \pm 0.8$ & $4.7 \pm 0.6$ & $4.4 \pm 0.6$ & $3.7 \pm 0.7$ & $3.4 \pm 0.6$ & $3.9 \pm 0.6$ & $3.4 \pm 0.6$ \\
& $\phi$ & $0.019 \pm 0.012$ & $0.001 \pm 0.021$ & $0.007 \pm 0.020$ & $0.006 \pm 0.028$ & $0.986 \pm 0.032$ & $0.982 \pm 0.026$ & $0.996 \pm 0.029$ \\
$f_{3}$ & $\mathrm{~A}$ & $5.2 \pm 1.0$ & $2.5 \pm 0.9$ & $2.9 \pm 0.8$ & $1.8 \pm 0.7$ & $2.7 \pm 0.8$ & $2.6 \pm 0.8$ & $2.1 \pm 0.8$ \\
& $\phi$ & $0.351 \pm 0.033$ & $0.356 \pm 0.055$ & $0.369 \pm 0.044$ & $0.249 \pm 0.057$ & $0.386 \pm 0.051$ & $0.379 \pm 0.048$ & $0.359 \pm 0.064$ \\
\hline \multicolumn{2}{r}{ Variance } & $72 \%$ & $57 \%$ & $58 \%$ & $46 \%$ & $43 \%$ & $51 \%$ & $42 \%$ \\
\multicolumn{2}{r}{ reduction } & & & & & & & \\
\hline
\end{tabular}

spectroscopic data is only 5 days and the frequency accuracy is only at $0.05 \mathrm{c} \mathrm{d}^{-1}$ level. The periodograms of the radial velocity and the first and second moment show a maximum near $0.7 \mathrm{c} \mathrm{d}^{-1}$ and all of its one-day aliases and so they are consistent with the star being a line-profile variable with frequency $f_{1}$ found in the multicolour photometry $\left(f_{1}\right.$ and $f_{2}$ are hardly resolved in the spectroscopic data). A phase plot of the radial velocity variation for $f_{1}$ is shown in Fig. 8. We find an average radial velocity of $21.4 \pm 0.5 \mathrm{~km} \mathrm{~s}^{-1}$ which is entirely compatible with the much less accurate value of $23 \mathrm{~km} \mathrm{~s}^{-1}$ listed in
Simbad. The amplitude of the fit to the radial velocity variation for $f_{1}$ shown in Fig. 8 is $2.8 \pm 1.0 \mathrm{~km} \mathrm{~s}^{-1}$. The peak-to-peak variation in the radial velocity amounts to $18 \mathrm{~km} \mathrm{~s}^{-1}$. No obvious periodicity is found in the equivalent width and the full-widthat-half-maximum.

We conclude to have found clear evidence for multiperiodic variations in the 7-colour Geneva photometry of HD 121190. Three significant frequencies are present. Each of them has amplitude ratios compatible with low-degree non-radial gravity-mode oscillations. The spectra are consistent with this 


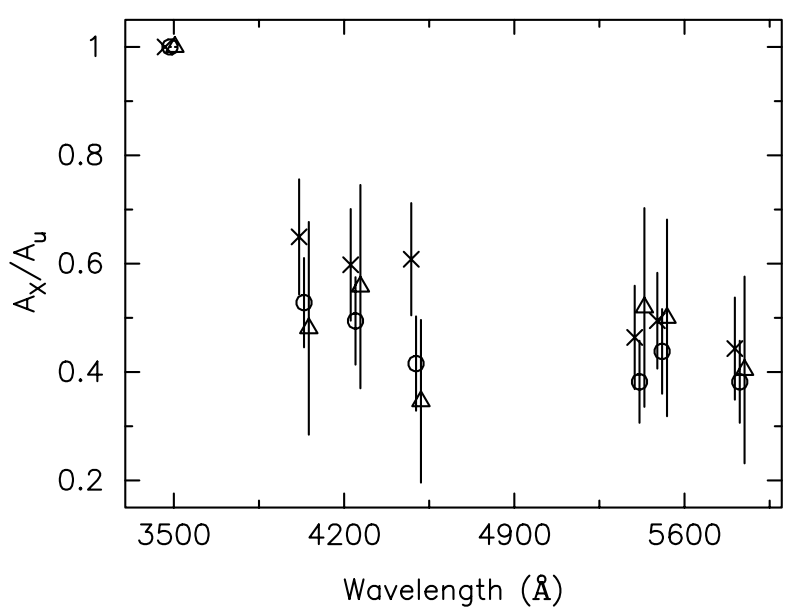

Fig. 7. Amplitude ratios $A_{X} / A_{U}$ for the three detected modes of HD 121190 with their errors, where $A_{X}$ stands for any of the amplitudes in the 6 Geneva filters $B_{1}, B, B_{2}, V_{1}, V, G$. The symbols are: $\times$ for $f_{1}, \circ$ for $f_{2}, \Delta$ for $f_{3}$. We have shifted the wavelengths of the filters artificially by $20 \AA$ in order to show the values for $f_{2}$ and by $40 \AA$ for $f_{3}$ for better visual inspection.

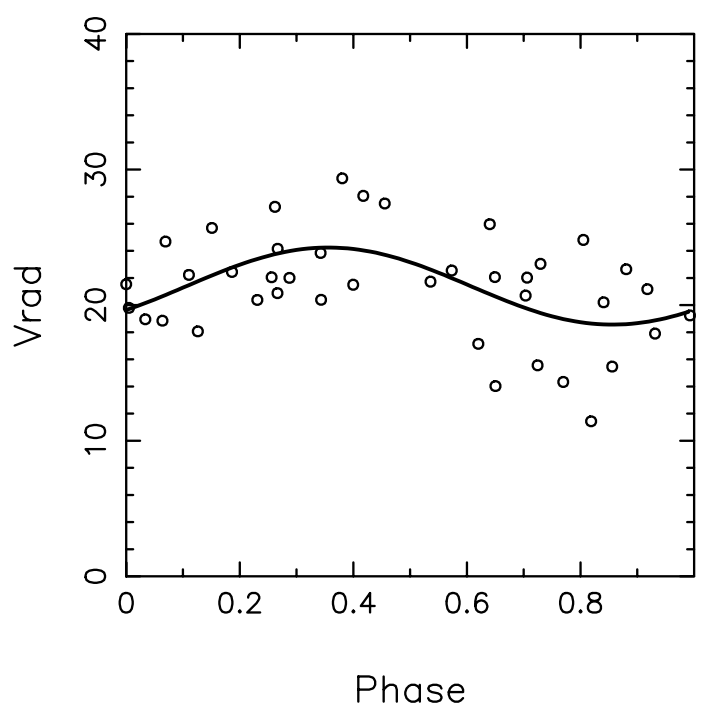

Fig. 8. The phase diagram of the radial velocity of HD 121190 derived from the profiles shown in Fig. 1. The open dots are the observations and the full line is a fit for the frequency $f_{1}$.

photometric variability. In view of its physical parameters we therefore classify HD 121190 as the coolest single and second most rapidly rotating SPB known to date.

\section{Discussion}

We have provided clear observational evidence for the occurrence of line-profile variability in two stars of spectral type B9. For one of these, HD 121190, we were able to detect three significant frequencies near $2.6 \mathrm{c} \mathrm{d}^{-1}$ in the multicolour photometry. The variability is consistent with high-order low-degree gravity-mode oscillations. For HD 106419 we were unable to unravel the complex low-amplitude frequency spectrum.

During the last half century, there has been quite some confusion and debate in the literature about the existence of a specific group of variables with spectral types between B7 V-III and A2 V-II and periods between 2 and $8 \mathrm{~h}$, baptised "Maia stars" by Struve (1955). We point out that these hypothetical stars would be partly situated within the SPB instability strip, extending towards the $\delta$ Scuti strip. Despite large search campaigns (see, e.g., Scholz et al. 1998, and references therein) clear detection of short-period variability for these stellar types remains ambiguous for most candidates. At the time when Struve (1955) made his suggestion, SPBs were still unknown. Applying the Struve (1955) criteria, HD 121190 would be classified as a Maia star. However, we object this type of classification. Indeed, ever since the observational studies by Waelkens (1991) and Waelkens et al. (1998) on the one hand, and the theoretical works by Dziembowski et al. (1993) and Gautschy $\&$ Saio (1993) on the other hand, it does not make sense any longer to speak of Maia stars for spectral types B. There is one clear physical mechanism that explains such oscillating stars: $\kappa$ driven, non-radial, gravity-mode oscillations, and so these objects should be termed SPBs. The short periods are sometimes claimed to be problematic from a theoretical viewpoint (e.g. Kallinger et al. 2002) but we would like to offer a different view. From their extensive study, Scholz et al. (1998) conclude that only 3 stars out of the 15 for which long-term observational studies are available are confirmed as periodic variables: $\gamma \mathrm{UMi}$ (A2III), $\gamma \mathrm{CrB}$ (A0V) and ET And (B9IV). These three stars have a $v \sin i$ of respectively 165,112 , and $80 \mathrm{~km} \mathrm{~s}^{-1}$. Hence they rotate much faster than the average SPB, just as our two target stars. As already discussed in Sect. 4.2, such rotation velocities imply that the effects of the Coriolis force may come into play and that this force introduces significant frequency shifts for the low-frequency gravity modes (Townsend 2003). We believe that such shifts offer the correct explanation for the relatively high observed frequency values for the modes that we have detected in HD 121190 and that Scholz et al. (1998) found for the three stars mentioned above. We also recall that theoretical instability strips so far have always been provided for non-rotating stars (e.g. Pamyatnykh 1999 and Fig. 2). The strip shown in Fig. 2 would no longer be placed in exactly the same position, and the periods of the oscillations would have a much broader allowed range if the Coriolis force would be taken into account in an accurate way.

As for the observational search for pulsating late-B to earlyA type stars, we warn that large high-precision multicolour photometric and/or high-resolution spectroscopic campaigns have to be undertaken before making any firm decisions about the presence or absence of low-amplitude multiperiodic oscillations, as also already emphasised by Scholz et al. (1998). One should not make firm conclusions based on short observing runs. Rather, dedicated campaigns of the type described in Aerts et al. (1999), De Cat et al. (2000), Mathias et al. (2001) and De Cat \& Aerts (2002) are needed to study such oscillations. The same is true for the gravity modes in the much cooler $\gamma$ Doradus stars (see, e.g., Poretti et al. 2002; Henry \& Fekel 2003; Aerts et al. 2004; Mathias et al. 2004 for some recent examples of such long-term campaigns).

Acknowledgements. We are much indebted to the referee, Dr. R. Townsend, for having pointed out some serious shortcomings in an 
earlier version of our paper. We thank our colleagues from the Institute of Astronomy of Leuven University who contributed to the gathering of the photometric data: Peter De Cat, Joris De Ridder and Gwendolyn Meeus.

\section{References}

Abt, H. A., Levato, H., \& Grosso, M. 2002, ApJ, 573, 359

Aerts, C., Cuypers, J., De Cat, P., et al. 2004, A\&A, 415, 1079

Aerts, C., De Cat, P., Peeters, E., et al. 1999, A\&A, 343, 872

Aerts, C., De Pauw, M., \& Waelkens, C. 1992, A\&A, 266, 294

Ausseloos, M., Scuflaire, R., Thoul, A., \& Aerts, C. 2004, MNRAS, 426, in press

Baade, D. 1989a, A\&AS, 79, 423

Baade, D. 1989b, A\&A, 222, 200

Breger, M., Stich, J., Garrido, R., et al. 1993, A\&A, 271, 482

Cuypers, J. 1987, The Period Analysis of Variable Stars, Academiae Analecta, Royal Academy of Sciences, Vol. 49, No. 3, Belgium

De Cat, P. 2002, in Radial and Nonradial Pulsations as Probes of Stellar Physics, ed. C. Aerts, T. R. Bedding, \& J. ChristensenDalsgaard, ASP Conf. Ser., 259, 196

De Cat, P., \& Aerts, C. 2002, A\&A, 393, 965

De Cat, P., Aerts, C., De Ridder, J., et al. 2000, A\&A, 355, 1015

De Cat, P., Briquet, M., Daszyńska-Daszkiewicz, Dupret, M.-A., et al. 2004, A\&A, submitted

Dupret, M.-A., De Ridder, J., De Cat, P., et al. 2003, A\&A, 398, 677

Dziembowski, W. A., Moskalik, P., \& Pamyatnykh, A. A. 1993, MNRAS, 265, 588
Gautschy, A., \& Saio, H. 1993, MNRAS, 262, 213

Henry, G. W., \& Fekel, F. C. 2003, AJ, 126, 3058

Heynderickx, D., Waelkens, C., \& Smeyers, P. 1994, A\&AS, 105, 447

Iglesias, C. A., \& Rogers, F. J. 1996, ApJ, 464, 943

Kallinger, T., Reegen, P., \& Weis, W. W. 2002, A\&A, 388, L37

Künzli, M., North, P., Kurucz, R. L., \& Nicolet, B. 1997, A\&AS, 122, 51

Mathias, P., Aerts, C., Briquet, M., et al. 2001, A\&A, 379, 905

Mathias, P., Le Contel, J.-M., Chapellier, E., et al. 2004, A\&A, 417, 189

Pamyatnykh, A. A. 1999, Acta Astr., 49, 119

Pamyatnykh, A. A., Handler, G., \& Dziembowski, W. A. 2004, MNRAS, 350, 1022

Poretti, E., Koen, C., Bossi, M., et al. 2002, A\&A, 384, 513

Rivinius, Th., Baade, D., \& Štefl, S. 2003, A\&A, 411, 229

Scargle, J. D. 1982, ApJ, 263, 835

Schaller, G., Schaerer, G., Meynet, G., \& Maeder, A. 1992, A\&AS, 96, 269

Scholz, G., Lehmann, H., Hildebrandt, G., Panov, G., \& Iliev, L. 1998, A\&A, 337, 447

Struve, O. 1955, Sky \& Telescope, 14, 461

Tassoul, J.-L. 1978, Theory of Rotating Stars (Princeton: Princeton University Press)

Townsend, R. H. D. 2002, MNRAS, 330, 855

Townsend, R. H. D. 2003, MNRAS, 343, 125

Waelkens, C. 1991, A\&A, 246, 453

Waelkens, C., Aerts, C., Kestens, E., Grenon, M., \& Eyer, L. 1998, A\&A, 330, 215 